KARL JANSEN - WINKELN

\title{
Zu einigen „Trinksprüchen“ auf ägyptischen Gefäßen ${ }^{1}$
}

Auf ägyptischen Gebrauchsgegenständen findet man seit dem Neuen Reich nicht selten kürzere, in Form einer Rede verfaßte Inschriften, die auf die Funktion dieses Gegenstandes Bezug nehmen². Eine Gruppe dieser Textchen, die Inschriften von fünf Weinamphoren aus Alabaster, sollen im folgenden behandelt werden, da ihr Inhalt z. T. nicht richtig verstanden worden ist. Drei der Gefäße stammen sicher, zwei mit hoher Wahrscheinlichkeit aus der 22./23. Dynastie ${ }^{3}$.

\section{Nr. 1}

Gefäß im Museo Arqueológico Provincial Granada Inv. Nr. 8319 aus dem (phönizischen) Grab Nr. 1 in Almuñécar aus der Zeit Takeloths II. Publiziert in:

Gamer-Wallert (1978), p. 24-30; Abb. 2; Taf. 1-3.

Padró I Parcerisa (1985), p. 64-70; pl. LXXVII-LXXX (mit ausführlicher Angabe der früheren Literatur auf p. 64).

Inschriftenband in Höhe der Henkel mit eingefügtem Kartuschenpaar: (Siehe S. 144)

\section{Zum hieroglyphischen Text:}

a) Gamer-Wallert und Padró I Parcerisa lesen $\bar{\equiv}$. Ein paar Gruppen weiter, in $p 3 w t j t 3 w j$, ist es fraglich, ob das obere Zeichen $\Longleftarrow$ oder $\rightleftharpoons$ zu lesen ist.

Das Zeichen über $\Longleftarrow$ ist zweifellos nicht das Ohr (Gardiner F 21), das höher und schmaler sein sollte, sondern das $\mathrm{km}$-Zeichen (I 6). Ganz ähnliche Formen findet man auf den zeitgenössischen Statuen Kairo CG 42225 und $42226^{4}$.

b) So; die obere Verlängerung des ff noch weit genug erhalten, um $f$, wie Gamer-Wallert und Padró I Parcerisa ergänzen, auszuschließen.

1 Häufig zitierte Literatur: Gamer-Wallert (1978): I. Gamer-Wallert, Ägyptische und ägyptisierende Funde von der Iberischen Halbinsel, Beihefte zum Tübinger Atlas des Vorderen Orients, B, 21, Wiesbaden 1978. Padró I Parcerisa (1985): J. Padró I Parcerisa, Egyptian-Type Documents from the Mediterranean Littoral of the Iberian Peninsula before the Roman Conquest, III, Etudes préliminaires aux religions orientales dans l'empire romain, 65, Leiden 1985. Äg. Biogr.: K. Jansen-Winkeln, Ägyptische Biographien der 22. und 23. Dynastie, Wiesbaden 1985 (ÄAT 8, 1/2).

2 Einige Beispiele bei Schott, Altägyptische Liebeslieder, Zürich 1950, „Dokumente“ $67 \mathrm{ff} .$, p. $122-3$. Vgl. auch unter den zahlreichen in den „Royal Cemeteries of Kush“ gefundenen Alabastergefäßen besonders Nr. 19-3-560 (D. Dunham, El Kurru [=RCK I], p. 31; 37, fig. 11j; pl. XXXIX. E/2), Nr. 16-4-74a (id., Nuri [=RCK II], p. 80; 83, fig. 54, pl. LXXIX), Nr. 21-2-396 und 397 (Dunham/Reisner, The West and South Cemeteries at Meroë [RCK V], p. 374; 375, fig. 202).

${ }^{3}$ Nr. 1 ist durch die Kartuschen datiert, Nr. 5 durch den Namen des zukünftigen Takeloth III., Nr. 4 durch den libyschen Namen des Vaters des Besitzers sowie den für diese Zeit typischen Titel „Königssohn des Ramses“; Nr. 3 enthält die Anrufung eines Königs Osorkon (s. d.). Auch Nr. 2 gehört nach Namen des Besitzers, Form der Hieroglyphen und Art des Textes mit größter Wahrscheinlichkeit in die Libyerzeit. Zu Alabastergefäßen im allgemeinen vgl. man die Ausführungen von G. Hölbl, Beziehungen der ägyptischen Kultur zu Altitalien, I, Leiden 1979, p. $240 \mathrm{ff}$.

${ }^{4}$ S. Äg. Biogr., p. 496, Z. 8 und p. 506, vorletzte Zeile. 

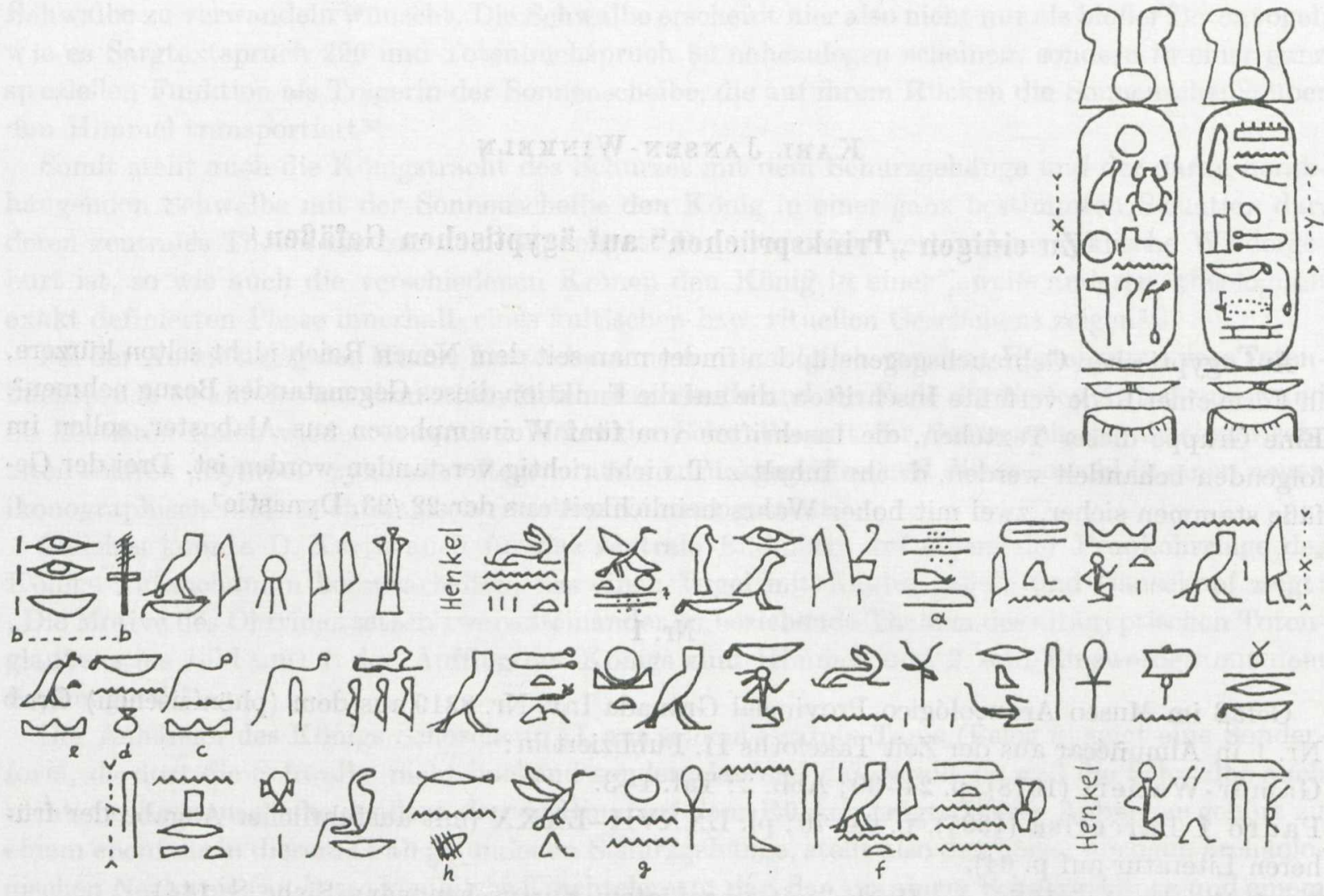

Gamer-Wallert liest unter dem Augenpaar $\mho^{5}$, Padró I Parcerisa richtiger $\circlearrowright$. Die scheinbare Verlängerung oben links ist wohl ein Loch im Stein (s. Gamer-Wallert, Taf. 2a).

c) So; Gamer-Wallert: - statt -

d) So; ungenaue Form bei Gamer-Wallert.

e) Das (klein geratene) $\triangle$ fehlt bei Padró I Parcerisa und bei Gamer-Wallert, die es in ihrer Anm. 49 (p. 26) aber richtig angibt.

f) Auf Taf. 3 bei Gamer-Wallert glaube ich ein $\triangle$ unter dem $n$ zu erkennen, das auch nach der Raumaufteilung dastehen sollte. Leider ist diese Stelle etwas verschmutzt. Gamer-Wallert und Padró I Parcerisa beide ohne 0 .

g) Auf derselben Tafel lese ich deutlich 으. Gamer-Wallert hat an der Stelle des zweiten $n$ einen Freiraum gelassen, Padró I Parcerisa gibt eine Zerstörung an.

h) Die Umrisse des Zeichens unter $m h n$ nicht mehr klar auszumachen (vgl. die Tafel bei GamerWallert). Es kann sich nur um ein 0 oder um ein $\triangle$ handeln.

\section{Übersetzung:}

„Ich bin aus meinem Fremdland gekommen, nachdem ich die Länder durchzogen habe und mir deine Riten vollzogen (1) worden sind, Urzeitlicher der Beiden Länder, der das Seinende geschaffen hat. Glanz geht von dir aus, der in allen Augen ist (2). Du sprichst aus die Luft des Lebens, die die Kehlen atmen läßt (3).

Ich bin in der 3 hjt (4), indem ich (über)voll bin mit den „Freuden“ von Bahriya und Dachla (5) durch das, was ich herbeibringe (6). Ein Brunnen der Gesundheit und des Lebens ist in mir, auf dessen Rand sich die $m h n n$-Schlange niederläßt (7).“

\footnotetext{
5 Ursprünglich hatte sie $\circlearrowright$ gelesen, vgl. Padró I Parcerisa (1985), p. 66 (e) mit n. 63.
} 
Anmerkungen zur Übersetzung:

1) $\mathrm{Zu} \mathrm{km}$ jrw „Riten vollziehen“ vgl. WB V, 128,17 sowie das vergleichbare $\mathrm{km}$ ` $b w$ in Äg. Biogr., p. 496-7, Z. 8-9. Die Frage ist, welche Riten hier gemeint sind. Es ist wohl so zu verstehen, daß es sich um ein durch irgendeine Zeremonie „geweihtes" Gefäß handelt.

2) Vgl. dazu Assmann, Liturgische Lieder an den Sonnengott, MÄS 19, 1969, p. 41-2 mit Anm. 14.

3) Daß die Stimme des Sonnengottes „,atmen läßt“ (u. ä.), ist ein ständiges Thema der Unterweltsbücher. Einige Beispiele bei Gamer-Wallert, p. 26-7.

4) Es ist klar, daß $3 h j t$ hier metaphorisch zu verstehen ist (vgl. WB I, 17, unter II), fraglich ist nur, wofür genau es steht. Da das Gefäß selbst der Sprecher ist (s. u.), kommen an dieser Stelle praktisch nur „Grab" und „Tempel“ in Betracht. „3hjt" als eine Bezeichnung des Grabes ist in dieser Zeit $\mathrm{m}$. W. nicht belegt, als eine Bezeichnung des Tempels bzw. des Allerheiligsten dagegen häufiger:

- Block Louvre C 258 (s. Daressy, RT 35, 1913, 130), Z. 7 ,den Weg einschlagen nach 3h-mnw, der 3hjt des ,Himmels"“

- dto., in derselben Zeile : „öffnen für ihn die Türflügel der 3hjt des Urzeitlichen der Beiden Länder“ - Statue Kairo CG 42225, c (s. Äg. Biogr., p. 495), Z. 2-3: „Amun ernannte mich zum Öffner der Türflügel des ,Himmels', so daß ich seine Gestalt sah, die in der 3hjt ist"

- dto., Z. 5: „Ich sah seine (des Sohnes) Schönheit als einen, der die 3hjt erleuchtet ..."

- dieselbe Statue, d, (op. cit., p. 497), Z. 11-12: „. . daß du öffnetest die Türflügel im Tor der $3 h j t^{\prime \prime}$

- Statue Kairo CG 42230, c (op. cit., p. 532), Z. 2 : „Ich erhielt Zutritt beim Gott als fähiger junger Mann und wurde eingeführt in die 3 hjt des ,Himmels " "

- Statue Kairo CG 42231, d (op. cit., p. 545), Z. 3: „Ich stieg auf der Treppe zur 3hjt des ,Himmels“ hinauf"

- Kartonage Cambridge Fitzwilliam Museum E 8.1896 (s. Quibell, The Ramesseum, Egyptian Research Account 2, 1898, pl. XXXa): „Der Prophet des Amonrasonther, der in den ,Himmel eintritt, der den sieht, der darin ist, der das Geheime kennt in der Abgeschiedenheit der 3hjt"

- Statue Kairo CG 42220, f, Z. 5-6 (s. Legrain, Statues et statuettes [Catalogue Général], III, 47): „Er [=Amun] beförderte meinen Sohn in meine Stellung in seinem Palast von Karnak, er leitete ihn in die ,3hjt der Beiden Herren'..."

Außerdem spricht die Tatsache, daß es sich um ein ,geweihtes" Gefäß handelt (vgl. Anm. 1) ebenfalls dafür, daß es für den Gebrauch im Tempel bestimmt war. Schließlich würde man, wäre es für eine Grabausstattung bestimmt gewesen, unbedingt den Namen des Besitzers in der Inschrift genannt haben. Das Fehlen irgendeiner konkreten Bezugsperson scheint mir das stärkste Argument dafür zu sein, daß mit 3hjt nur ein Tempel gemeint sein kann.

5) Damit ist natürlich Wein gemeint.

$\underline{t} t f$ ließe sich ebensogut als Partizip $\underline{t} t f(t)$ übersetzen : „die $3 h j t$, die überflutet ist“.

Zu den Oasennamen vgl. J. O sing in: Mélanges Gamal Eddin Mokhtar, Kairo 1985, Vol. II, p. 179 ff. 6) Gamer-Wallert und Padró I Parcerisa übersetzen — haben daran weitergehende Spekulationen geknüpft (es soll hier „,im Gefolge des Sonnengottes“ bedeuten). Die Deutung als Relativform in der gut belegten Bedeutung „herbeibringen, darbringen“ ist sehr viel unkomplizierter.

7) Dieser letzte Zusatz ist zwar grammatisch klar, aber man fragt sich, was genau damit gemeint sein mag. Gamer-Wallert und Padró I Parcerisa vermuten, daß an die Schutzfunktion der Schlange zu denken sei, und das ist zweifellos die nächstliegende Deutung, obwohl der Zusatz nicht recht zum Kontext zu passen scheint. An eine Erklärung der $M \not h n$-Schlange als Symbol der Verjüngung und Erneuerung des Gefäßinhalts zu denken ist wohl zu spekulativ.

Aus dem letzten Satz der Inschrift, ,ein Brunnen der Gesundheit und des Lebens ist in mir" geht in aller Deutlichkeit und ohne jeden Zweifel hervor, daß hier das Gefäß spricht ${ }^{6}$, und dazu paßt der

6 Wie auch in Text Nr. 2, s. u. 
gesamte Tenor und Inhalt des übrigen Textes sehr gut. Einer Erläuterung bedürfen allenfalls die einleitenden Sätze jjj.n.j $m$ ḩsst.j phrr.n.j $t 3 w$. Sie erklären sich wohl daher, daß der Wein aus den Oasen nach Ägypten gekommen ist, wie der Text ja ausdrücklich angibt, und das Gefäß sich auf diese „Reise“ bezieht.

Es handelt sich also um ein Weingefäß für den Tempelkult, das in diesem Text selbst als fiktiver Sprecher von der Herkunft (des Weins), „Weihung“ und Zweckbestimmung berichtet.

Padró I Parcerisa, p. $66 \mathrm{ff}$. hat diese Inschrift in höchst phantasievoller Weise interpretiert. Er hält das Gefäß für ein zum „Export“ bestimmtes Weingefäß und den Sprecher der Inschrift für den dieses Gefäß abholenden Handelspartner. Mit dem im Text apostrophierten Sonnengott sei in Wirklichkeit der Pharao gemeint (Takeloth II., dessen Kartuschen ja in das Inschriftband eingefügt sind). Es lohnt sich wohl nicht, diesen Deutungsversuch im einzelnen zu widerlegen.

Nr. 2

Gefäß in Privatbesitz; in Spanien nordöstlich von Cádiz gefunden. Publiziert in :

Gamer-Wallert (1978), p. 81-5 mit Abb. 21; Tafel 22-24 (mit Angabe der früheren Literatur auf p. 82 Anm. 82).

Inschriftenband in Höhe der Henkel:

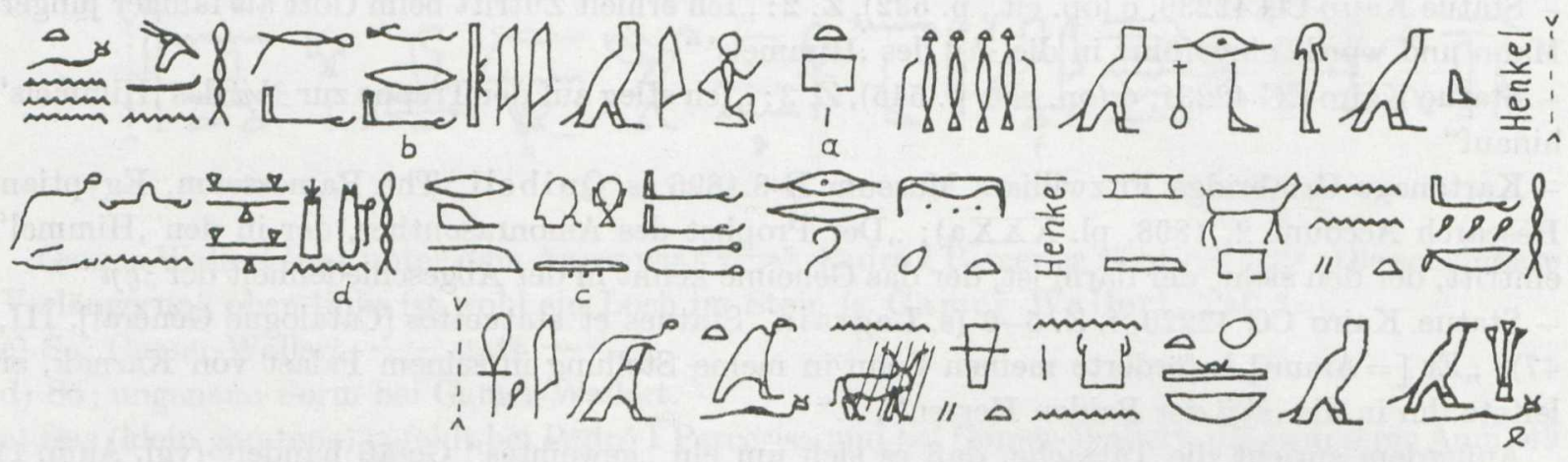

Zum hieroglyphischen Text:

a) Auf dem Photo (Taf. 22b) nicht ganz sicher, aber sehr viel wahrscheinlicher bei Gamer-Wallert.

b) Das obere Zeichen zweifellos ein $d$; eine etwas andere Form weiter unten in $\underline{d} d$.

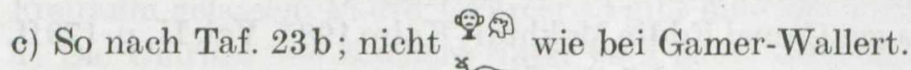

d) Auf dem Photo (Taf. 24a) nicht ganz deutlich; besonders das (unübliche) „Anhängsel“ rechts von Y unsicher.

$\mathrm{Zu}$ der Kombination zweier $h \mathfrak{h}$-Zeichen verschiedener Form in einem Wort vgl. Vernus in: Karnak VI, p. 219(a).

e) Der Stellung der Zeichen nach ist es durchaus möglich, das . $f$ vor dem darüber stehenden $h 3$ zu lesen.

Übersetzung:

„Die Art, Gutes zu tun aus mir (1) und die Sorgen (2) zu vertreiben: Benetze deinen Leib mit dem, was im Innern des „Sees der Wahrheit“ ist! (3)

,Gold', mögen handeln deine Arme auf Erden für alle Zukunft (4): Er soll keinen Mangel äußern an allen Dingen (5), für den Ka des zm3̈tj des Kamutef DDd-Hrw-jw.f- $n h$. ." 
Anmerkungen zur Übersetzung:

1) Dem ganzen Zusammenhang nach (s. u.) kann hier nur etwas wie ,aus mir, aus meinem Innern“, d. h. aus dem Gefäß selbst gemeint sein; das $\square$ gehört daher als Determinativ zu hnt. Bei der darauf folgenden Gruppe scheint eine Emendation zu $\langle n\rangle . j-j m j j$ unvermeidlich (so auch schon bei Gamer-Wallert, p. 82 Anm. 86). Der Strich unter $\square$ ist entweder ein Raumfüller, so daß dann $m$-hnt $\langle n\rangle$.j-jmjj , aus mir" zu lesen wäre, oder aber - und dies erscheint mir wahrscheinlicher - er

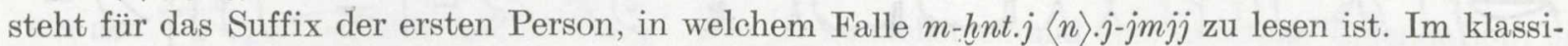
schen Mittelägyptischen stehen Suffixe und (attributives) $n=-j m j j$ zwar in komplementärer Distribution ${ }^{7}$, im spätzeitlichen Mittelägyptischen scheint dies aber nicht mehr so zu sein, vgl.

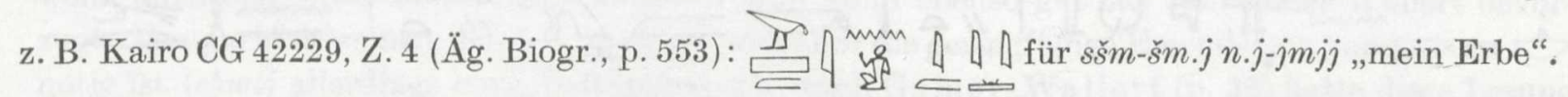

Man könnt schließlich noch an eine ganz andere Möglichkeit denken, bei der sich sogar die Emendation zu $\langle n\rangle . j-j m j j$ erübrigte: jmij könnt hier dazu dienen, die Präpositionalphrase $m$-hnt.j zu „adjektivieren“, analog zu $n . j-j m j j$, wo die Präpositionalphrase $n . j$ durch $j m j j$ zu einem Adjektiv gemacht wird (wie auch immer dieses Syntagma ursprünglich gebildet worden sein mag). Ein solcher Gebrauch von jmij (mit anderer Präpositionalphrase) wäre allerdings ganz singulär, und so möchte ich diesen Vorschlag auch nur unter größtem Vorbehalt machen.

2) Sicher so (vgl. WB II, 120,17-8) und nicht „Säbelantilope“ wie bei Gamer-Wallert, p. 82-3. Der Antilopenkopf ist hier nur ein „phonetisches Determinativ“. Das $\longleftarrow$ als Ersatz von ist in dieser Zeit ungemein beliebt. Beispiele aus Äg. Biogr., Text A 1, b,1; $5 ; 6 ; 9 ; 10 ; c, 5 ; 6 ;$ A 5 , d,5; $9[2 \times] ;$ A 7, d,12; A 11, c; A 15, d,9; A 17, b,3; A 18, c,6; 10; A 20, b,3; d.

Mit $d r m h$ endet der erste Abschnitt des Textes: Es handelt sich praktisch um die Überschrift zum nächsten Satz, der seinerseits eine Art „Gebrauchsanweisung“ für das Gefäß ist. Statt „die Art, Gutes zu tun ... "könnte man auch freier „wie man Gutes tut" übersetzen.

3) $\langle m-\rangle \underline{h} n w m r m 3^{\ulcorner} t$ zu lesen. Eine vergleichbare Schreibung von $(m-) \underline{h} n w(\widehat{X})$ ) aus dieser Zeit auf der Statue Kairo CG 42210 (s. Äg. Biogr., p. 463 und p. 70 [11]).

Mit dem „See der Wahrheit“ ist natürlich das Gefäß gemeint. Man kann nicht umhin, an „in vino veritas" $\mathrm{zu}$ denken.

4) Was immer dieser Satz besagen soll, hier ist natürlich von Hathor als Göttin der Trunkenheit die Rede (vgl. z. B. LÄ II, 1027; Daumas in: RdE 22, 1970, 75f.).

5) Zweifellos $n n$ dd d.f $h 3 m$ jht $n b t$ zu lesen; so schon bei Gamer-Wallert auf Anregung von H. Brunner.

Der Text enthält also folgende Elemente:

Eine „Gebrauchsanweisung“ für den Krug samt Überschrift, die sich direkt (in der 2. Sg. mask.) an einen nicht weiter spezifizierten Benutzer des Gefäßes richtet;

eine Bitte an Hathor als Göttin des Rausches in einer sehr allgemein gehaltenen Formulierung, die vermutlich besagen soll, daß die Göttin dafür sorgen möge, daß der Krug nicht leer wird; die Nutzanwendung daraus: der am Ende erstmals genannte Besitzer des Gefäßes soll keinen Mangel leiden.

\section{Nr. 3}

Gefäß im Museo Arqueológico Provincial Granada Inv. Nr. 8329 aus dem (phönizischen) Grab Nr. 15 in Almuñécar. Publiziert in:

Gamer-Wallert (1978), p. 30-34; pl. 4.

Padró I Parcerisa (1985), p. 84-90; pl. CVI-VII (mit Angabe der früheren Literatur auf p. 85).

\footnotetext{
7 S. Satzinger, ZÄS 113, 1986, 150 ff., besonders p. 153.
} 
Inschriftenband in Höhe der Henkel:

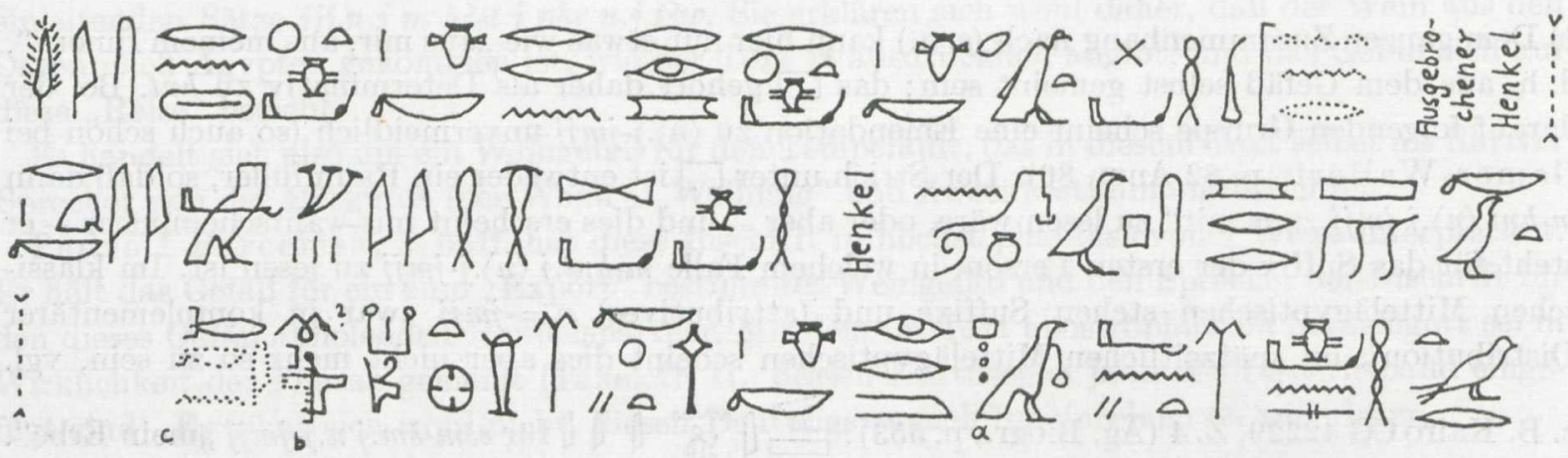

Zum hieroglyphischen Text:

a) Padró I Parcerisa gibt $\square$ statt $\square$ (wie richtig bei Gamer-Wallert).

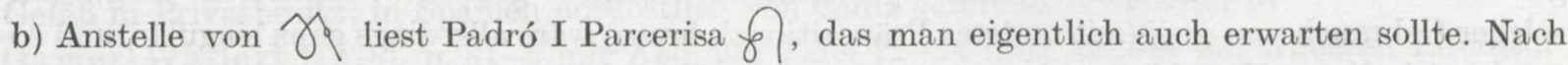
den Photos ist keine sichere Entscheidung möglich. Die Vertauschung von $\gamma$ und $\varnothing$ ist in dieser Zeit durchaus möglich, vgl. meine Äg. Biogr., p. 56 (50); J $\in$ A 38, 1952, pl. XI, Z. 28; Piankoff, Mythological Papyri, Nr. 19, Z. 19 v. r.

c) Die publizierten Photos erlauben leider keine genauere Bestimmung des Ausmaßes des Ausbruchs.

\section{Übersetzung:}

„Möge Mut dein Herz [nicht] zurückhalten (1) zu trinke nund zu tun, was dein Herz wünscht. Trinke und preise Amun (2), indem du angenehm machst die ,Trockenheit' (3): Das ist es, was sie liebt. Berausche dich, flechte Kränze, gib (sie) an deinen Kopf (4), salbe (dich) für sie mit Myrrhen, handle nach deinem Herzen, Herr der Doppelkrone in Bubastis, Ka des Osorkon."

\section{Anmerkungen zur Übersetzung:}

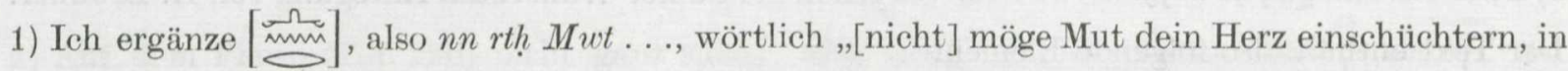
Furcht versetzen vor ...". Zum Verb $r$ th vgl. WB II, 460, besonders unter Nr. 4, wo $r$ th im Gegensatz zu sj3m gebraucht wird, das auch hier im nächsten Satz erscheint.

Mut, auf die sich auch die folgenden femininen Pronomina beziehen, wird hier offenbar wegen ihrer Verbindung mit Hathor genannt, vgl. Bonnet, Reallexikon der ägyptischen Religionsgeschichte, p. 492.

2) Ganz unsicher; nur als Verlegenheitsvorschlag möchte ich $m(n)$ (Imperativ) „preisen“ lesen (vgl. WB II, 435,10 und Gardiner, Notes on the Story of Sinuhe, p. 37), rnn ebenfalls ohne Determinativ (allerdings mit zwei $n$ ) findet sich auf Gefäß Nr. 4 . Wie immer die Stelle zu verstehen ist, den Vorschlag von Padró I Parcerisa, p. 87(b), die Gruppe $\overbrace{m}$ als $r n h h$ zu verstehen, halte ich für ganz unmöglich.

3) D. h. dich betrinkst. Das Verständnis von $\triangle$ भि ist ebenfalls äußerst unsicher. Es ist wohl kaum $w \check{s}(r) t$, mit Umstellung des $\triangle$, zu lesen, sondern das $\triangle$ wird einfach überflüssig sein, ebenso wie in wäre. Die Schreibung ohne $r$ ist problemlos erklärbar. 
4) $j m j$ hier in der seltenen Konstruktion ohne Objekt.

5) Der Schluß (? s. u.) der Inschrift ist zweifellos der problematischste Abschnitt. Sowohl GamerWallerts Deutung „... in Bubastis ist der Ka des Osorkon“ als auch die von Padró I Parcerisa "protect in Bubastis the Ka of Osorkon" sind nach den ägyptischen Wortstellungsregeln nicht möglich.

Padró I Parcerisa möchte das $〕$ nach $j b . k$ als dittographisch streichen und erklärt $\int_{0 \mid l}$ als ein aus $\int_{f} \delta$ entstelltes $n \underline{d}$,schützen“. Das ist zunächst einmal eine unnötige Emendation, da es ja auch ein Verb nd $\underline{d} t j$,schützen“" gibt (WB II, 375). Aber die Lesung $n \underline{d}(t j)$ ist keineswegs sicher, wenn auch epigraphisch durchaus möglich. Man kann ebenso gut das von Gamer-Wallert bevorzugte $s h m$ darin erkennen. Ich lese $n b s h m t j$, wozu nur die geringfügige Emendation von $\circlearrowright \mathrm{zu}$ nötig ist (shmtj allerdings ohne Determinativ!); auch Gamer-Wallert (p. 32) hatte diese Lesung schon erwogen.

Der Hauptgrund für meine Übersetzung ist die Einbindung dieser Stelle in den übrigen Text. Irgendwelche Aussagen über einen Osorkon in Bubastis oder auch Bitten für ihn, die nichts mit den vorhergehenden Aufforderungen zur Festfreude zu tun haben, sind hier doch gar nicht zu erwarten. Die Erwähnung eines Osorkon kann eigentlich nur heißen, daß sich die Aufforderungen an ihn richten 8 .

Liest man $n b$ shmtj, kann es sich natürlich nur um einen König Osorkon handeln. Die Schreibung eines Königsnamens ohne Kartusche ist in dieser Zeit nicht ohne Parallele, vgl. Montet, Les constructions et le tombeau de Psousennès à Tanis, Paris 1951, p. 92, Z. $3 ; 17$.

Man muß zugeben, daß dies alles sehr merkwürdig ist. Das ist diese Stelle, vor allem das $k 3 n+$ Namen ohne jedweden Titel, aber bei jeder Interpretation!

Welche Deutung man auch vorziehen mag, der Name Osorkon, im Zusammenhang mit Bubastis genannt, legt auf jeden Fall eine Datierung in die 22./23. Dynastie nahe, und es gibt nichts in der Inschrift, was diesem Ansatz widerspräche.

\section{Nr. 4}

Gefäßbruchstück Kairo CG 18435; Fundort unbekannt9. Publiziert in :

Von Bissing, Steingefässe (Catalogue Général), Wien 1907, p. 83 (mit Nachtrag auf p. XLVI) (ohne Photo).

\section{Übersetzungen :}

Gamer-Wallert (1978), p. 33-4.

S. Schott, Altägyptische Liebeslieder, Zürich 1950, p. 130, Nr. 93. Zum Titel und Namen des Besitzers vgl. auch:

G. Maspero, Momies royales de Deir el-bahari, Paris 1889, p. 719.

Gauthier, in: ASAE 18, 1919, 256-7.

Redford, in: JAOS 93, 1973, 9 n. 50.

Inschriftenband in Höhe der Henkel ${ }^{10}$ :

\footnotetext{
8 Es ist darum auch möglich, daß der Text mit $n b$ shmtj beginnt, der Vokativ also am Anfang steht, wodureh allerdings der Text nicht mehr direkt nach dem Gefäßhenkel beginnen würde; vgl. dazu das Gefäß Nr. 4, bei dem die Henkel ebenfalls keine „textgliedernde“ Funktion haben (anders allerdings bei Nr. 3 und 5 !).

${ }_{9}^{9}$ Gamer-W allert (1978), p. 33-34 (mit Anm. 131) sagt allerdings, das Gefäß sei in der Cachette von Deir el-Bahari gefunden worden. Dafür gibt es m. W. keinerlei Hinweis. In Masperos Publikation der Cachette ist es nur als Beleg für einen Titel zitiert worden, nicht, weil es dort gefunden worden wäre!

10 Die Wiedergabe des Textes basiert nur auf den Publikationen von v. Bissing und Maspero, nicht auf Photos wie bei den Gefäßen Nr. 1-3 und 5 !
} 


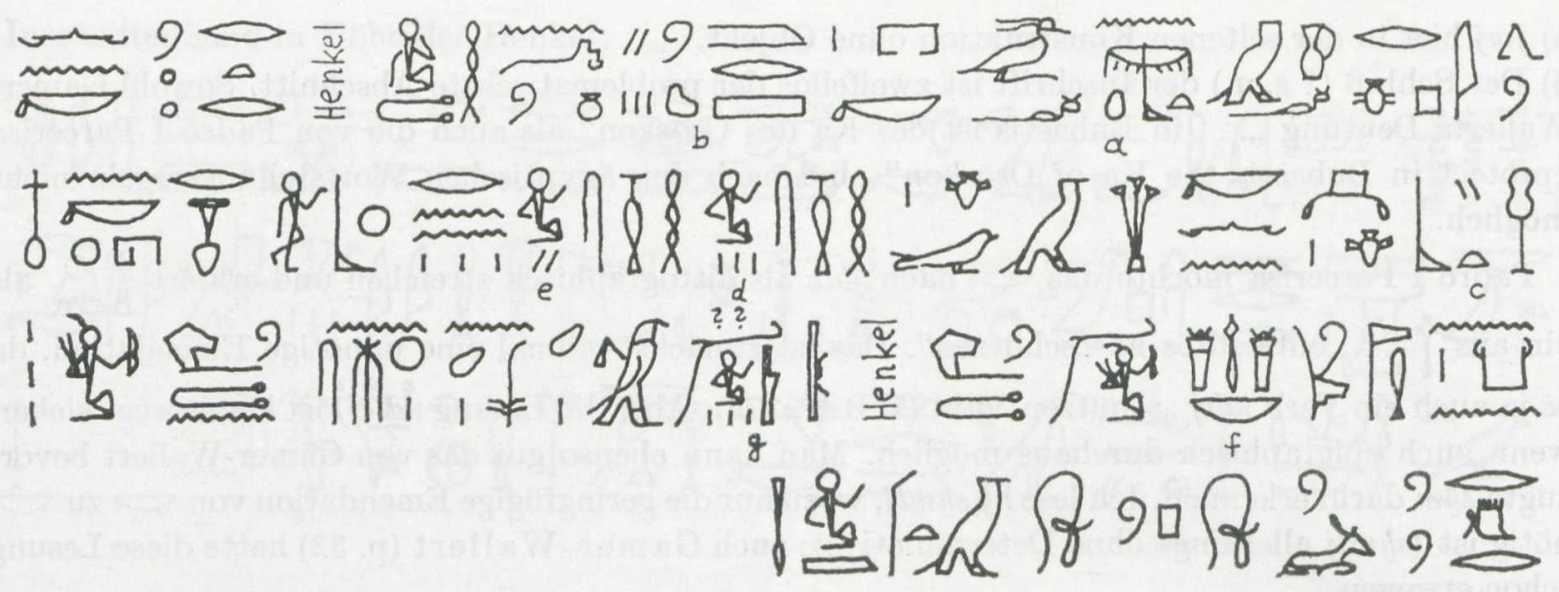

Zum hieroglyphischen Text:

a) So im CG; im Original zweifellos ein Krug.

b) Wohl (ohne Emendation) so zu lesen. Der CG gibt $" 1$ ? (p. XLVI) als " " ?. Wie aus der ganzen Abschrift hervorgeht, hatte v. Bissing allerdings wenig von dem Text verstanden. Die Verlesung von $\square \mathrm{zu} \rightleftharpoons$ ist daher leicht verständlich.

c) So der CG. Die besondere Form des $3 b$-Zeichens ist schon möglich (ähnlich etwa auf Kairo CG 42220, c, 6 [eigene Abschrift]), aber das $\triangle$ ist sehr zweifelhaft. $3 b$,aufhören “ ist in dieser Zeit sonst

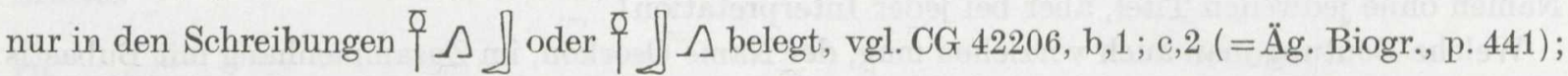
CG 42210, d,2 (=op. cit., p. 464); CG 42225, c,8 (=op. cit., p. 495); CG 42220, c,6; Statue Karnak T 35 (s. Karnak-Nord IV, fig. 143, unten, Z. 1); CG 42254, links, Z. 5 (unpubl., eigene Abschrift). Es ist nicht ausgeschlossen, daß v. Bissing das $\Lambda$ in 0 verlesen hat. Auch das $\backslash$ (Richtung!) darüber ist merkwürdig.

d) Determinativ im CG durch „sic“ bestätigt.

e) So im CG; ob richtig?

f) So ursprünglich im CG, im Nachtrag (p. XLVI) dann in f ",verbessert“. Bei nicht ganz sauber geschnittenen Hieroglyphen sind sich $\{$ und fo in dieser Zeit sehr ähnlich. Aufgrund von Parallelen (z. B. PSBA 13, 1890, 36) zweifellos \& zu lesen, obwohl auch Maspero, loc. cit. J liest.

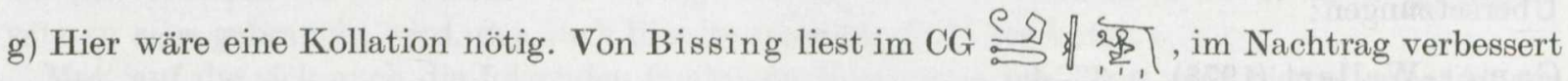

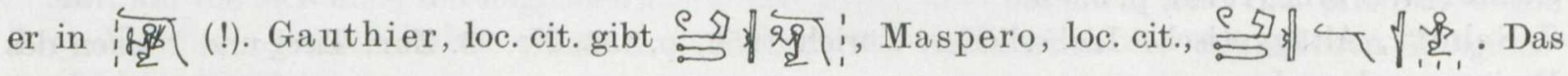
merkwürdige ff nach ḩ3wtj scheint also sicher, obwohl es da nichts verloren hat. Der Titel nach $h 3$ wtj kann aber unmöglich $m r m \check{s}$ sein, denn dieser wird ja unmittelbar vor ḩ3wtj aufgeführt! Deshalb ist vielleicht Masperos Wiedergabe vorzuziehen und $m r j z w t$,Truppenvorsteher" zu lesen, obwohl ich dazu in dieser Zeit keine Parallele wüßte. In der Inschrift am Grab Osorkons II.11 führt ein $P$ 3-šrj-n-3st den Titel $m r m \check{s}$ wr nз jzwt n $\breve{S} m^{2} w$ Mḥ.

\section{Übersetzung}

„Der Wein möge kommen, er geselle sich zum Bier (1). Er möge dein Haus mit Freude überfluten, so daß du Tag und Nacht trunken bist ohne aufzuhören (2). Sei fröhlich ohne Kummer! Sänger sollen singen (3), jubeln, tanzen, um dir einen Festtag zu bereiten, für den Ka des Propheten der

\footnotetext{
11 S. Montet, Les constructions et le tombeau d'Osorkon II à Tanis, Paris 1947, pl. XXII-III.
} 
Bastet, der Großen der Herrin von Bubastis, des General, Kommandeurs und Truppenvorstehers (4) Hrw, des Sohnes des „Königssohnes des Ramses“, des Kommandeurs der gesamten Truppen Jwpwt, gerechtfertigt."

Anmerkungen zur Übersetzung:

1) Zu nbtjs. Fairman, ZÄS 91, 1967, 7 und Äg. Biogr., p. 545, Z. 11. Hier ist natürlich nicht etwa gemeint, daß sich der Wein im selben Gefäß mit Bier vermischt, sondern er soll bei Gelagen zusätzlich zu Bier getrunken werden.

2) Wörtl. „indem es nicht ein Aufhören für dich gibt“.

3) $h s w w\langle r\rangle h s(t)$

4) S. o. Anmerkung g.

\section{Nr. 5}

In Assur gefundenes, von Asarhaddon in Sidon erbeutetes Gefäß. Publiziert (nur Photos und Übersetzung) in:

Von Bissing, Zeitschrift für Assyriologie 46, NF 12, 1940, 155-9, Abb. 8a/b.

Gamer-Wallert (1978), p. 42-4; Taf. 8-10.

$\mathrm{Zu}$ dem sekundär angebrachten keilschriftlichen Text s. R. Borger, Die Inschriften Asarhaddons, Königs von Assyrien, Beihefte AfO Nr. 9, Graz 1956, p. 8.

Inschriftenband in Höhe der Henkel mit eingefügtem Kartuschenpaar:
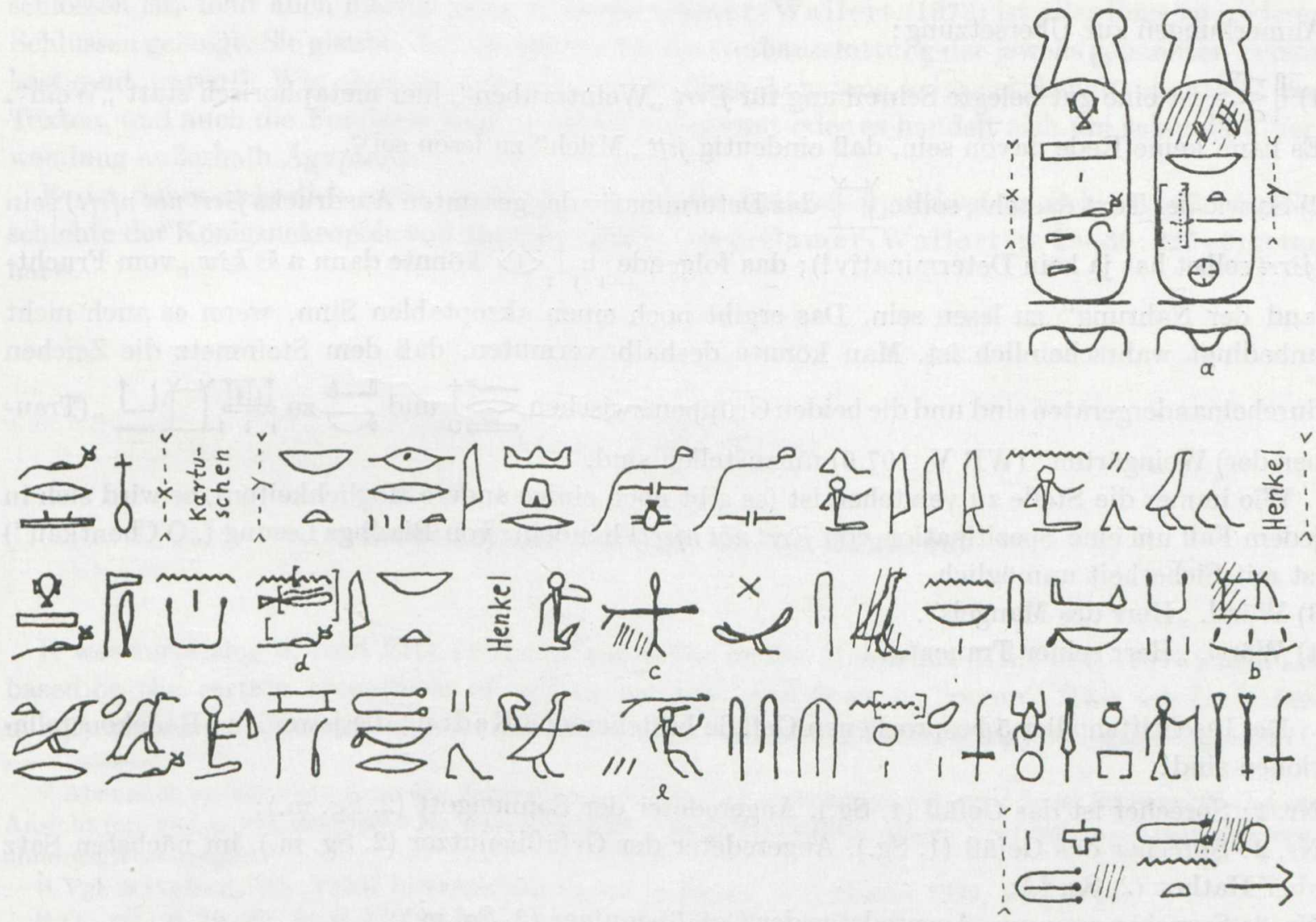

Zum hieroglyphischen Text:

a) Das oberste Zeichen kann ich - trotz vorhandener Reste - nicht ergänzen.

Ein Rext von $\gamma_{r}$ ist noch sichtbar. Es ist nicht ausgeschlossen, daß hinter $\gamma$ noch ein hat. 
b) Das obere Zeichen ziemlich verwischt, aber die Lesung scheint sicher.

c) $\infty$ und \& zu einem Monogramm vereint ist ziemlich ungewöhnlich. Die Vertiefung links unten ist wohl nur eine Beschädigung des Steins.

d) Die Zeichenspuren sind zwar nicht besonders signifikant, erlauben aber ohne weiteres die Lesung $\leftrightharpoons$ Ob unter dem $d$ noch ein (flaches) Zeichen stand, läßt sich nach dem Photo nicht schlüssig beurteilen. Für ein an sich zu erwartendes reicht der Platz nicht.

e) Eine sehr merkwürdige Form des Ideogramms für m̌̌c, das gemeint sein muß. Möglicherweise trug der dargestellte Krieger noch eine zweite Feder am Hinterkopf. Gleichfalls bemerkenswert die Pluralstriche I/I.

\section{Übersetzung:}

Kartuschen:

Harsaphes, der Herrscher der Beiden Ufer, [?] der Herr von Herakleopolis.

Haupttext:

„Sei mir willkommen, der (du) kommst 〈aus〉 Bahriya mit allen guten Trauben (1) der Weingärten (? ?) (2). Mögest du sie geben dem Bedürftigen (3), dem Bekümmerten, dem Trauernden (4), für den Ka des Propheten des Harsaphes, Königs der beiden Länder, des Großen von Pr-shm-hpr-r, des „Königssohnes des Ramses“, des Truppenkommandeurs und Befehlshabers Takeloth; seine Mutter ist die erste Haremsdame Tnts3, gerechtfertigt."

\section{Anmerkungen zur Übersetzung:}

1) $4 \overbrace{\Delta \| 11}^{\infty}$ ist eine gut belegte Schreibung für $j 3 r r t$ „Weintrauben“, hier metaphorisch statt „Wein“. Es kann keine Rede davon sein, daß eindeutig $j r \underline{t} t$, „Milch“ zu lesen sei12.

2) So wie der Text dasteht, sollte $Y \mathcal{K}$ das Determinativ des gesamten Ausdrucks j3rrt nbt nfr(t) sein (j3rrt selbst hat ja kein Determinativ!); das folgende $L_{1} \longleftrightarrow$ könnte dann $n \check{s} 3 k 3 w$,vom Fruchtland der Nahrung" zu lesen sein. Das ergibt noch einen akzeptablen Sinn, wenn es auch nicht unbedingt wahrscheinlich ist. Man könnte deshalb vermuten, daß dem Steinmetz die Zeichen

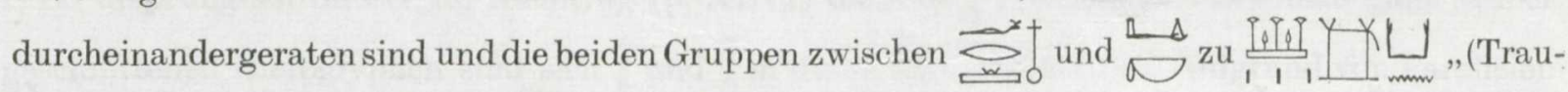
ben der) Weingärten“ (WB V, 107,6) umzustellen sind.

Wie immer die Stelle zu verstehen ist (es gibt noch einige andere Möglichkeiten), es wird sich in jedem Fall um eine Spezifikation von j3rrt nbt nfr(t) handeln; von Bissings Lesung („,O Chentkau“) ist mit Sicherheit unmöglich.

3) Wörtl. „Herr des Mangels“.

4) Wörtl. „Herr seiner Trauer“.

Die Inschriften aller 5 besprochenen Gefäße bestehen aus Reden; die jeweiligen Redekonstellationen sind:

Nr. 1: Sprecher ist das Gefäß (1. Sg.), Angeredeter der Sonnengott (2. Sg. m.);

Nr. 2: Sprecher das Gefäß (1. Sg.), Angeredeter der Gefäßbenutzer (2. Sg. m.), im nächsten Satz aber Hathor (2. Sg. f.);

Nr. 3: Sprecher anonym, Angeredeter der Gefäßbenutzer (2. Sg. m.);

Nr. 4: dieselbe Konstellation wie Nr. 3;

Nr. 5: Sprecher der Gefäßbenutzer (1. Sg.), Angeredeter das Gefäß (2. Sg. m.).

12 So von Bissing, op. eit., p. 157, Anm. 2 und Gamer-Wallert, p. 43, Anm. 201, die allerdings auch die Lesung j3rrt erwägt. 
Der Text von Nr. 1 schildert Schicksal und Funktion des Gefäßes selbst; keine konkrete Person wird erwähnt. In Nr. 2 fordert das Gefäß den Benutzer auf zu trinken (mit einer zusätzlichen Bitte an Hathor, den Gefäßbesitzer keinen Mangel leiden zu lassen), in Nr. 3 und 4 fordert ein anonymer Sprecher, in dem man wieder das Gefäß selbst vermuten darf, den Nutzer zu Trunk und Festfreude auf. Unterschiedlich ist dabei allerdings der Anschluß der Bezugsperson: In Nr. 4 durch $n k 3 n$ (Titel und Name), in Nr. 3 direkt durch Anrede im Vokativ. Der Text von Nr. 5 schließlich enthält eine Aufforderung an das Gefäß, Traurigen, Bedürftigen usw. zu trinken zu geben.

Für die (ursprüngliche) Verwendung der Gefäße kann man folgendes daraus schließen: Nr. 1 war mit Sicherheit ein Tempelgefäß; anders ist die Anrede an Gott, das Fehlen einer konkreten Person und der Passus über das „Vollziehen der Riten“ nicht zu erklären.

Alle anderen werden Weingefäße zum Gebrauch der Le benden gewesen sein $^{13}$ : In Nr. 2 wird davon gesprochen, „die Sorgen zu vertreiben“, in Nr. 5, den „Bedürftigen, Bekümmerten, Trauernden“ zu trinken zu geben, in Nr. 3 und 4 vom Trunk bei festlichen Gelegenheiten. Der Name der Gefäßbesitzer hat in keinem einzigen Fall den Zusatz $m 3^{c}-h r w$. Gefäß Nr. 5 gehört zudem mit größter Wahrscheinlichkeit dem zukünftigen König Takeloth III., der zum Zeitpunkt der Abfassung dieser Inschrift also noch nicht den Thron bestiegen hatte ${ }^{14}$.

Nicht genauer erschließen läßt sich natürlich, wo und wann im einzelnen diese Gefäße benutzt wurden, ob sie bei allen Gelegenheiten als Weinkrüge dienten oder nur bei besonders festlichen Zeremonien, etwa den Totenfesten.

Es ist unwahrscheinlich, daß auch nur eines der Gefäße primär als Teil der Grabausstattung gefertigt war. Obwohl eine sekundäre Verwendung als Grabbeigabe natürlich keineswegs ausgeschlossen ist, fehlt auch hierfür jeder Hinweis. Gamer-Wallert (1978) ist allerdings zu anderen Schlüssen gelangt. Sie glaubt, daß die Gefäße für die Grabausstattung der jeweils genannten Person bestimmt waren 15. Wie oben gezeigt, gibt es für diese Annahme keinerlei Anhaltspunkte in den Texten, und auch die Fundorte sind entweder unbekannt oder es handelt sich um sekundäre Verwendung außerhalb Ägyptens.

Es ist daher sicherlich nicht angängig, aus diesen Gefäßen irgendwelche Schlüsse für die Geschichte der Königsnekropole von Tanis zu ziehen, wie es Ga mer-Wallert (p. 29-30; 225-6) getan hat 16 .

13 Aber auch andere nicht-funeräre Verwendungsweisen sind nicht ausgeschlossen : So ist Gamer-Wallerts Ansicht (op. cit., p. 44), das Gefäß Nr. 5 sei eine Weihgabe an Harsaphes, dessen Kartuschen es trägt, gewesen, ohne weiteres möglich.

14 Vgl. Kitehen, The Third Intermediate Period in Egypt, Warminster 1972, § 76, p. 96.

15 Op. eit., p. 29 [Nr. 1], p. 33 [Nr. 3 und 4], p. 84 [Nr. 2]. Nr. 5 hält sie aber für eine Weihgabe, s. die vorletzte Fußnote.

16 Erst recht bieten die Gefäßtexte keinerlei Stütze für Padró I Parcerisas abenteuerliche Thesen: Der Inschrift von Nr. 1 entnimmt er, es sei für den Export bestimmt gewesen (s. o., zu Nr. 1), Gefäß Nr. 3 soll einem ,in die Mysterien der Hathor Eingeführten“ gehört haben (op. eit., p. 88) etc. 